\title{
Photonic generation of picosecond pulse doublets from the nonlinear splitting of super-Gaussian pulses
}

\author{
Christophe Finot \\ Laboratoire Interdisciplinaire CARNOT de Bourgogne, UMR 6303 UBFC-CNRS, 2100 Dijon, France
}

\begin{abstract}
We experimentally demonstrate the generation of temporal pulse doublets from the propagation of initial super-Gaussian waveforms in an optical fiber with anomalous dispersion. The nonlinear focusing dynamics leads to picosecond structures that are characterized both in amplitude and phase. Their close-toGaussian Fourier-transform limited shape is found in excellent agreement with numerical simulations. This single-stage reshaping scheme is energy efficient, can sustain $\mathrm{GHz}$ repetition rates and temporal compression factors around 10 are demonstrated.
\end{abstract}

\section{Introduction}

Generation of picosecond optical pulse doublets at high repetition rates remains a technological challenge. Indeed, the optoelectronics bandwidth required to generate these double pulses is much higher than the one usually required to synthesize an isolated waveform. Therefore, all-optical approaches are fully relevant to overcome the electronics bottleneck and to provide optical doublets with a duration below $10 \mathrm{ps}$. In this context, several linear solutions have relied on photonic first-order derivation [1] using long-period fiber gratings, fiber Bragg gratings or on-chip microdisk resonators. Nonlinear approaches in optical fibers can also be advantageous thanks to the combination of the dispersive and Kerr effects. Indeed, numerous examples of doublets have been reported in fiber lasers. However, their repetition rates are typically a few tens of MHz only. Cavity-free solutions that do not require mode-locking processes also offer attractive solutions and several strategies have been explored such as chirp-induced splitting of pulses [2], pulse copying thanks to an external triangular waveform [3] or the compression of higher-order solitons.

In this contribution, we introduce a new solution based on the nonlinear propagation of a super-Gaussian pulse in a fiber with anomalous dispersion. This problem, also known as the box-problem or the dam-break problem, has recently stimulated a renewed interest in optics, mainly driven by the theoretical study of the nonlinear dynamics of the temporal $[4,5]$ or spatial $[6,7]$ coherent structures that emerge upon propagation. We show here experimentally that it can find practical applications. Indeed, a pair of Gaussian-like pulses emerge during the evolution of an initial flattop pulse in a single-mode waveguide with a focusing nonlinearity [8].

\section{Experimental setup}

We have implemented an experiment sketched in Fig. 1 based on devices that are commercially available and typical of the telecommunication industry. An external cavity laser at $1550 \mathrm{~nm}$ emits a highly-coherent continuous wave that is intensity modulated with a lithium niobate modulator to obtain super-Gaussian pulses at a repetition rate of $2 \mathrm{GHz}$. The initial pulses have an initial duration of $42 \mathrm{ps}$, are highly symmetric and present a flat plateau and sharp edges so that it approximates the ideal box waveform (see dashed grey line in Fig. 3a). Pulses are amplified by an erbium-doped fiber amplifier before nonlinear propagation in a set of four identical $900 \mathrm{~m}$ long optical SMF-28 fibers with second-order dispersion of $20.10^{-3} \mathrm{ps}^{2} / \mathrm{m}$ and a nonlinear Kerr coefficient of 1.1 $/ \mathrm{W} / \mathrm{km}$. In order to prevent the building of unwanted stimulated Brillouin backscattering, we have inserted three optical isolators. The characterization of the output pulse properties is ensured by a complex optical spectrum analyzer (Apex Technologies) that enables us to get access to both the temporal phase and intensity profiles with a temporal resolution below 1 ps.

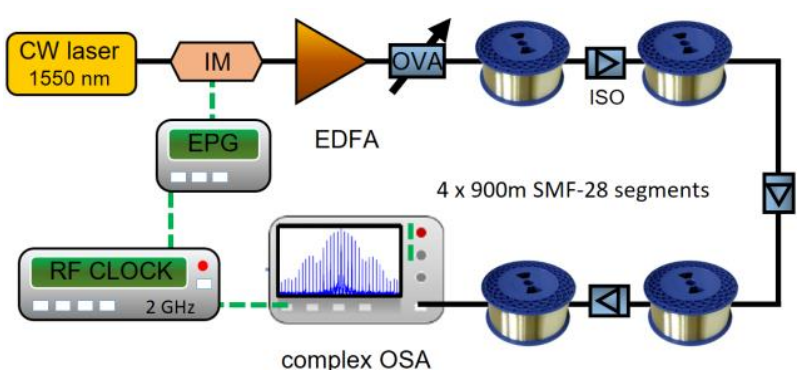

Fig. 1. Experimental setup. CW : continuous wave; IM : intensity modulator; EPG : electronic pattern generator; EDFA : erbium doped fiber amplifier; ISO : isolator ; OVA : optical variable attenuator.

\footnotetext{
* Corresponding author: christophe.finot $@$ u-bourgogne.fr
} 


\section{Experimental results}

Panels 1 of Fig. 2 summarize the experimental evolution of the intensity and phase profiles as a function of the average input power. When increasing the integrated nonlinearity in the fiber with anomalous dispersion, the initial super-Gaussian waveform reshapes into two wellseparated pulses. For additional propagation or nonlinearity, a third temporal component emerges from the central part of the resulting waveform. The experimental amplitude and phase features are both reproduced closely by numerical simulations of the propagation based on the nonlinear Schrodinger equation taking into account the losses of the fiber and the exact input conditions (see panels 2).

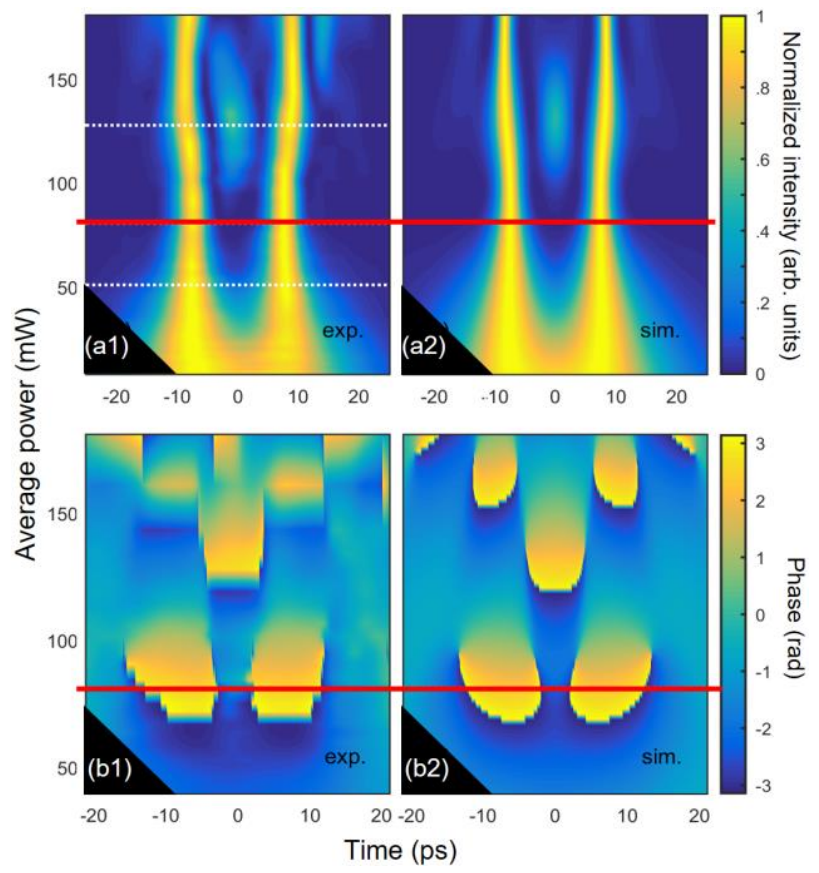

Fig. 2. Evolution of the output temporal intensity and phase profiles (panels a and $b$, respectively) of the waveform as a function of the initial average power for a fixed propagation length of $3.6 \mathrm{~km}$. Experimental results (panels 1) are compared with numerical simulations (panels 2). Intensity profiles are normalized by the peakpower. The red horizontal line strands for the results detailed in Fig. 3.

A more detailed view of the temporal profile obtained for an average power of $80 \mathrm{~mW}$ (corresponding to an input peak power of $1 \mathrm{~W}$ and a pulse energy of $40 \mathrm{pJ}$ ) is proposed in Fig. 3. For this power, two identical picosecond pulses are generated and spaced by $15.2 \mathrm{ps}$. The output pulse structures have a fwhm duration of 4.3 ps (i.e a compression factor of 9.8 with respect to the input waveform) and an excellent extinction ratio is observed. Once again, the experimental results are in remarkable agreement with numerical simulations. They confirm that the temporal phase profile over the two pulses is very flat and that the two pulsed parts are in-phase. This contrasts with other methods such as optical derivation where the leading and trailing pulses are $\pi$-phase shifted [1]. A better understanding of the nonlinear dynamics will stimulate further theoretical research with more advanced tools. Using topological perspective will enable to better determine the theoretical guidelines to apply and the balance between the duration, peak power, dispersion/diffraction and nonlinearity that has to be targeted [7].

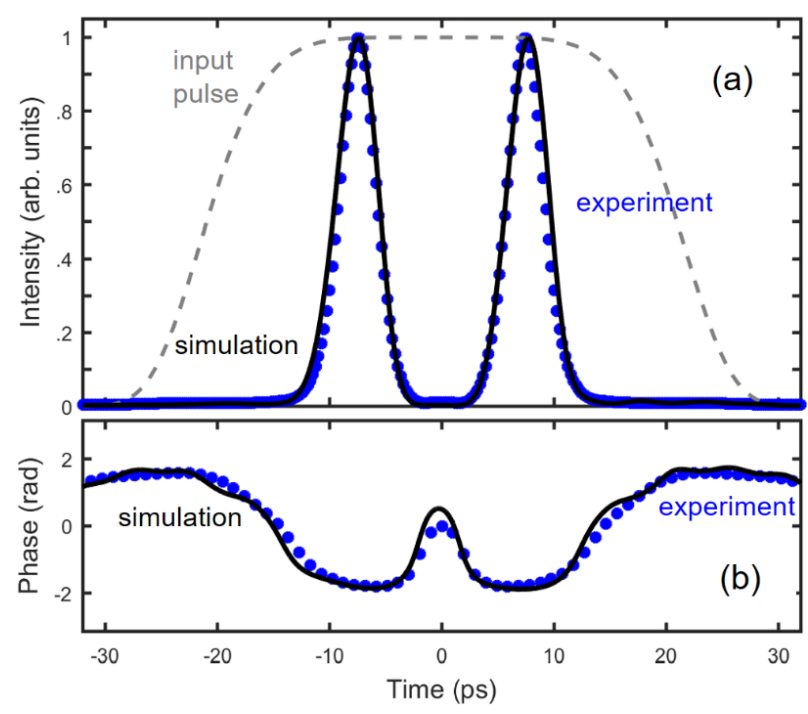

Fig. 3. Details of the temporal intensity and phase profiles (subplots a and b, respectively) obtained for an input average power of $80 \mathrm{~mW}$. Experimental results (solid black lines) are compared with numerical simulations of the NLSE (blue full circles). The input intensity profile is plotted with a dashed grey line.

\section{References}

1. R. Slavík, Y. Park, M. Kulishov, R. Morandotti, and J. Azaña, Opt. Express 14, 10699-10707 (2006).

2. L. Helczynski, B. Hall, D. Anderson, M. Lisak, A. Berntson, and M. Desaix, Phys. Scr. T84, 81 (2000).

3. N. Verscheure and C. Finot, Electron. Lett. 47, 11941196 (2011).

4. F. Audo, B. Kibler, J. Fatome, and C. Finot, Opt. Lett. 43, 2864-2867 (2018).

5. G. A. El, E. G. Khamis, and A. Tovbis, Nonlinearity 29, 2798 (2016).

6. W. Wan, D. V. Dylov, C. Barsi, and J. W. Fleischer, Opt. Lett. 35, 2819-2821 (2010).

7. G. Marcucci, D. Pierangeli, A. J. Agranat, R.-K. Lee, E. DelRe, and C. Conti, Nat. Commun. 10, 5090 (2019).

8. C. Finot, Laser Phys. Lett. 17, 025103 (2020). 